July 1999 • NREL/TP-500-26371

\title{
Conversion of Phase II Unsteady Aerodynamics Experiment Data to Common Format
}

M.M. Hand

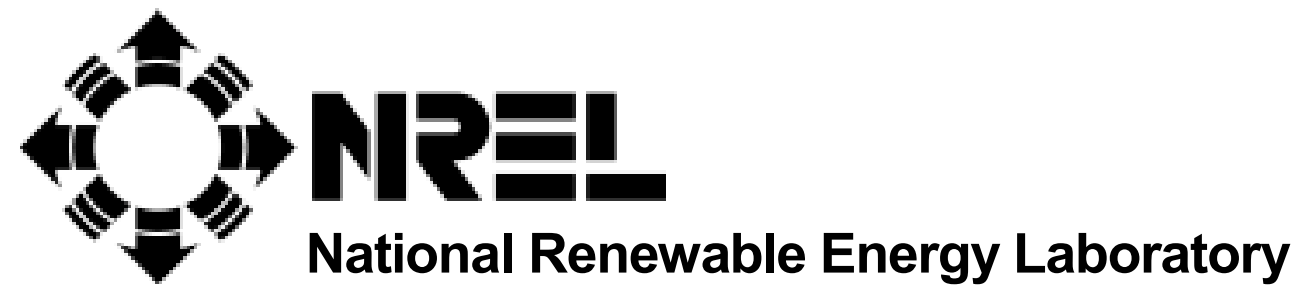

1617 Cole Boulevard

Golden, Colorado 80401-3393

NREL is a U.S. Department of Energy Laboratory

Operated by Midwest Research Institute • Battelle • Bechtel

Contract No. DE-AC36-98-G010337 
July 1999 - NREL/TP-500-26371

\title{
Conversion of Phase II Unsteady Aerodynamics Experiment Data to Common Format
}

\author{
M.M. Hand
}

Prepared under Task No. WE901110

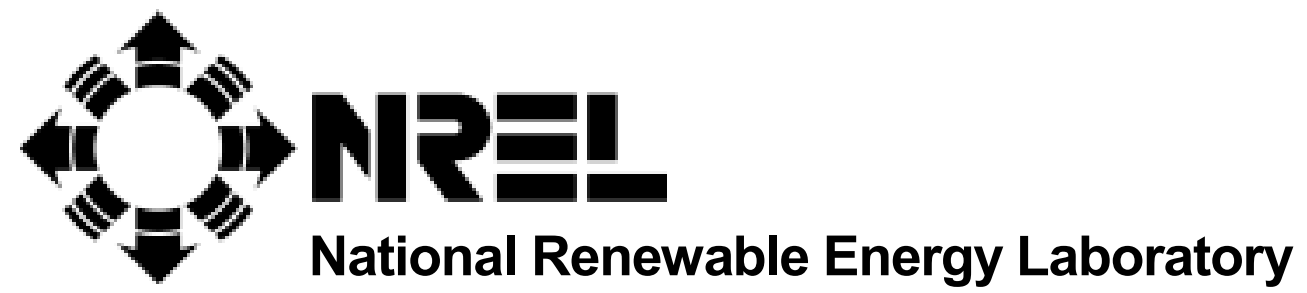

1617 Cole Boulevard

Golden, Colorado 80401-3393

NREL is a U.S. Department of Energy Laboratory

Operated by Midwest Research Institute • Battelle • Bechtel

Contract No. DE-AC36-98-G010337 


\section{NOTICE}

This report was prepared as an account of work sponsored by an agency of the United States government. Neither the United States government nor any agency thereof, nor any of their employees, makes any warranty, express or implied, or assumes any legal liability or responsibility for the accuracy, completeness, or usefulness of any information, apparatus, product, or process disclosed, or represents that its use would not infringe privately owned rights. Reference herein to any specific commercial product, process, or service by trade name, trademark, manufacturer, or otherwise does not necessarily constitute or imply its endorsement, recommendation, or favoring by the United States government or any agency thereof. The views and opinions of authors expressed herein do not necessarily state or reflect those of the United States government or any agency thereof.

Available to DOE and DOE contractors from:

Office of Scientific and Technical Information (OSTI)

P.O. Box 62

Oak Ridge, TN 37831

Prices available by calling 423-576-8401

Available to the public from:

National Technical Information Service (NTIS)

U.S. Department of Commerce

5285 Port Royal Road

Springfield, VA 22161

$703-605-6000$ or $800-553-6847$

or

DOE Information Bridge

http://www.doe.gov/bridge/home.html

Printed on paper containing at least $50 \%$ wastepaper, including $20 \%$ postconsumer waste 


\title{
CONVERSION OF PHASE II UNSTEADY AERODYNAMICS EXPERIMENT DATA TO COMMON FORMAT
}

\author{
M. Maureen Hand \\ National Wind Technology Center \\ National Renewable Energy Laboratory \\ 1617 Cole Blvd., Golden, CO 80401
}

\begin{abstract}
A vast amount of aerodynamic, structural, and turbine performance data were collected during three phases of the National Renewable Energy Laboratory's Unsteady Aerodynamics Experiment (UAE). To compare data from the three phases, a similar format of engineering unit data is required. The process of converting Phase II data from a previous engineering unit format to raw integer counts is discussed. The integer count files can then be input to the new postprocessing software, MUNCH. The resulting Phase II engineering unit files are in a common format with current and future UAE engineering unit files. An additional objective for changing the file format was to convert the Phase II data from English units to SI units of measurement.
\end{abstract}

\section{Nomenclature}

$\begin{array}{ll}q & \text { Estimated dynamic pressure, from psi to } \mathrm{Pa} \\ \rho & \text { Air density, from slug/ } \mathrm{ft}^{3} \text { to } \mathrm{Pa} / \mathrm{m}^{3} \\ r & \text { Radial distance from center of hub, from in. to } \mathrm{m} \\ \omega & \text { Rotational speed, from } \mathrm{rad} / \mathrm{s} \text { to } \mathrm{rpm} \\ V & \text { Wind speed, } \mathrm{m} / \mathrm{s} \\ c f & \text { Centrifugal force, from } \mathrm{psi} \text { to } \mathrm{Pa} \\ C_{p} & \text { Pressure coefficient } \\ \text { Counts } & \text { Integer value of raw data sample } \\ \text { slope } & \text { Calibration coefficient } \\ \text { offset } & \text { Calibration coefficient } \\ \alpha & \text { Angle of attack corrected for upwash, deg } \\ \text { lfa } & \text { Local flow angle, deg } \\ \text { new } & \text { Converted integer count value } \\ \text { old } & \text { Original engineering unit value }\end{array}$

\section{Introduction}

The National Renewable Energy Laboratory has completed Phase IV of the Unsteady Aerodynamics Experiment. A highly instrumented downwind Horizontal Axis Wind Turbine has provided a wealth of aerodynamic, structural, and turbine performance data to researchers studying issues such as the effect of unsteady aerodynamic events on wind turbine performance. Large quantities of data were collected for two rotor configurations: untwisted blades (Phase II) and twisted blades (Phase III and Phase IV). These three phases of testing produced two different engineering unit formats. The Phase II, untwisted-blade data were stored on optical disks in engineering unit form. A total of 239 channels were stored, 190 of which were measured data, 5 were time channels, and the rest were derived channels added during the post-processing process. The post-processing procedure converted integer counts produced by the data 
acquisition system to engineering units. In addition, the procedure corrected pressure measurements for centrifugal force effects, normalized blade surface pressures and upwash corrected angle of attack flag measurements. The low-speed shaft azimuth angle data were smoothed to account for sensor limitations, and a number of errors were introduced during this process (Miller et al., 1994).

When the Phase III data were collected, a new method of post-processing the data was implemented. This program, MUNCH, is easily adaptable and has been used in subsequent processing of data collected from the unsteady aerodynamics experiment. To provide consistency among all the unsteady aerodynamics experiment data, the Phase II engineering unit files were converted to integer count data similar to that originally produced with the instrumentation. This was accomplished by reversing the operations performed in producing the engineering unit files. These integer count data files were then input to MUNCH. This produced output engineering unit files that are now in a consistent format with the Phase III and Phase IV engineering unit files.

\section{Procedure}

Originally, the pressure tap measurements were stored as normalized pressure coefficients. The raw integer count value was converted to engineering units using the slope and offset calibration coefficients. A centrifugal force correction based on the radial location of the tap was added to the engineering unit value. This value was then normalized with the estimated dynamic pressure. These formulae follow:

$$
\begin{gathered}
q=\frac{1}{2} \rho\left[(r \omega)^{2}+V^{2}\right] \\
c f=\frac{1}{2} \rho(r \omega)^{2} \\
C_{p}=\frac{\text { Counts } * \text { slope }+ \text { offset }+c f}{q}
\end{gathered}
$$

The wind speed and air density values represent averages over the 5-min. data campaign. The rotational frequency was assumed to be constant at $72 \mathrm{rpm}$ or $7.54 \mathrm{rad} / \mathrm{s}$. The conversion process simply reversed this procedure by solving for Counts. This was done in two steps. First, the dynamic pressure and centrifugal force corrections were removed. Next, slope and offset effects were eliminated in a loop for all channels at once.

The total pressure probe channels also had undergone the centrifugal force correction. This centrifugal force amount was subtracted from the dynamic pressures stored in the original data files prior to the elimination of slope and offset effects.

The upwash correction applied to the local flow angle measured by the flags follows:

$$
\alpha=(-5.427 E-5) * l f a^{3}+(6.713 E-3) * l f a^{2}+0.617 * l f a-0.8293
$$

This equation resulted from curve-fitting data measured in a wind tunnel (Butterfield et al., 1992). The known angle of attack measured in the wind tunnel was plotted versus the local flow angle measured by the flag. The third order curve fit is shown above. When this data was plotted on opposite axes, a curve fit provided the following equation that was used to remove the upwash correction from the angle of attack flag data. The local flow angles that resulted from 
this equation were converted to integer count values through reversal of the slope and offset operations.

$$
l f a=(2.5542 E-4) * \alpha^{3}-.021454 * \alpha^{2}+1.6692 * \alpha+.87154
$$

The time channels were copied directly from one format to the other using a slope of 1 and an offset of 0 because they were already in integer format (except for the milliseconds channel). The milliseconds channel was in decimal format and required a slope of $1000 \mathrm{~ms} / 4095$ counts to be converted to an integer. The low-speed shaft azimuth angle channel was treated similarly, with a slope of $360^{\circ} / 4095$ counts and an offset of 0 . The errors introduced in the azimuth angle data during the original processing remained after the conversion.

The conversion program, included in Appendix A, first reads the header file associated with the original data. This file contains the slope and offset values used to convert counts to engineering units. It also contains the average values for barometric pressure and air temperature used to determine air density and the average wind speed necessary to calculate the estimated dynamic pressure. Next, the program reads the first record containing all 239 channels into memory. The program loops through each channel individually to determine if a correction is to be removed. If the channel represents a pressure tap, the centrifugal force and dynamic pressure corrections are removed. If the channel represents a total pressure probe, the centrifugal force correction is removed. If the channel represents an angle of attack flag, the upwash correction is removed. Once all corrections are removed, each channel is then converted to a 2-byte integer as follows:

$$
\text { new }=\operatorname{int}\left(\frac{\text { old }- \text { offset }}{\text { slope }}\right)
$$

The new record is written to output. This is done for each record recorded during the 5-min. campaign, resulting in more than 150,000 records. This new data file is stored as raw data on compact disk using the original tape filename with a '.dat' extension.

A new header file was also produced as output. This file contains the slope and offset values for each channel. All of the slopes and offsets for the pressure channels, total pressures, barometric pressure, and absolute pressure were converted to Pascals from psi or mb for consistency with the Phase III data format. The following conversions were used: $6894.8 \mathrm{~Pa} / \mathrm{psi}, 100 \mathrm{~Pa} / \mathrm{mb}$. The blade number order was changed to be consistent with subsequent phases of the experiment. Looking upwind, the instrumented blade is blade 3, followed by blade 1 and blade 2 in the clockwise direction.

These two output files were then used as input to the MUNCH program which produces engineering unit files. A comparison was made between the MUNCH-processed engineering unit file and the original engineering unit file on a sample-by-sample basis for each channel. If, for a given sample, the difference between the new and old values was outside the range \pm 0.00024 * old, a counting variable was incremented. (The 12-bit resolution of the PCM streams indicates that a difference of 1 bit in 4095 will introduce an error of \pm 0.00024 . This range is applicable for all channels except pressure-related measurements, angle of attack flags $[ \pm 0.1$ degrees--Butterfield et al., 1992], azimuth angle [ \pm 0.088 degrees or 360/4095], and milliseconds [ \pm 0.244 or $1000 / 4095])$. The maximum difference was also recorded. This procedure was performed on several campaigns, and an example is included in Appendix B.

The channels compare favorably, with little or no difference between the engineering units processed from the converted files and the engineering units created with the original processing 
procedures. The pressure measurements cannot be compared because they were normalized differently. The angle of attack measurements are within 0.028 degrees of each other, which is within the resolution of the flag at 0.1 degrees. The local meteorological measurements were converted exactly from one format to the other except for the wind elevation measurements. Because the maximum elevation angle difference introduced is less than 0.0008 degrees, this difference was considered negligible. The azimuth angle channel has a resolution of 0.87 , which is greater than the maximum difference recorded. The strain gage measurement differences exceed the limit at values very close to zero. This does not happen frequently, and is negligible when comparing fractions of Newton-meters. The maximum difference between the millisecond measurements is less than the resolution of .244 ms.

Another comparison was made between the 5-min. mean, maximum, and minimum values that were stored in the original header files and those stored in the newly created header files. An example of this comparison is included in Appendix C. The total pressure channels are very close on average, so the differences observed on a sample by sample basis were attributed to fluctuations in rotor speed and air density. The original centrifugal force correction originally was based on a constant rpm and 5-min. average air density. The current processing uses an instantaneous rpm and air density, which results in different centrifugal force corrections.

The normalized pressures were used in MUNCH to determine normal and tangential force coefficients at each span location. The baseline data were established by using methods discussed in Simms et al. 1996, and were plotted against angle of attack. This resulted in a plot consistent with that produced before the conversion process and provides confidence in the conversion of the normalized pressure coefficients.

\section{Summary}

The vast amount of data collected from Phases II, III, and IV of NREL's Unsteady Aerodynamics experiment is stored on compact discs in a common engineering unit format. The Phase II data were successfully converted to integer counts and reprocessed with the current software, MUNCH. Thus, data comparisons can easily be made using software common to all phases of the experiment.

\section{References}

Butterfield, C.P., W.P. Musial, and D.A. Simms (1992): “Combined Experiment Phase I Final Report”, NREL/TP-257-4655, National Renewable Energy Laboratory, Golden, CO.

Miller, M.S., D.E. Shipley, M.C. Robinson, M.W. Luttges (1996): "Determination of Data Reliability for Phase II of the Combined Experiment", NREL/TP-442-6914, National Renewable Energy Laboratory, Golden, CO.

Simms, D.A., M.C. Robinson, M.M. Hand, L.J. Fingersh (1996): “A Comparison of Baseline Aerodynamic Performance of Optimally-Twisted Versus Non-Twisted HAWT Blades", Proceedings of 15th ASME Wind Energy Symposium, Houston, TX. 


\section{Appendix A}

FORTRAN Code for Conversion Program 
program convert

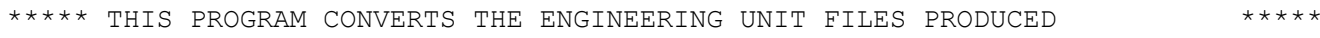

$\star \star \star \star *$ DURING PHASE II TESTING BACK TO COUNTS IN ORDER TO PROCESS

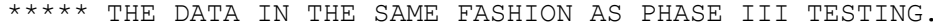

$\star \star \star * \star$ WRITTEN BY: MAUREEN HAND

$\star \star \star * *$ LIST OF VARIABLES

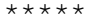

$\star \star \star \star \star *$ adens

$\star \star \star * \star$ aoa $(4)$

air density average over $5 \mathrm{~min}$. data set

$\star \star \star \star * \star *$

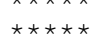

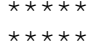

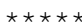

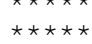

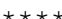

PCM numbers of angle of attack flags

$\star * * * * \mathrm{AZ}$

$\star * \star * *$ baro

air temperature average over $5 \mathrm{~min}$. data set

$\star \star \star * \star * *$ index of azimuth angle channel

$\star * \star * * *$

Barometric pressure average over 5 min. data set $\star_{\star \star *} * *$

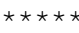

$\star \star \star \star \star$ cardmask() sets high end bits to indicate card change

$\star \star \star \star * \star *$

list of channel descriptions required for new header $* \star \star \star *$

$\star * * * *$ chanid() list of 8 character channel id codes required in new header**

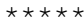

centrifugal force

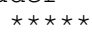

$\star \star \star \star \star *$ count

$\star \star \star \star \star *$ disk

$\star \star \star * \star$ disks

$\star \star \star * *$ diskstr

current disk being processed

names of disks to be processed

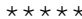

$\star \star \star * *$ header

$\star \star \star \star * \star$ IB

$\star \star \star * *$ infil

name of header file

$* * * * *$ instr

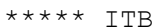

$\star \star \star \star * *$ newrec ()

$\star \star \star * *$ NUMCHAN

$\star \star \star \star \star *$ NUMPRESS

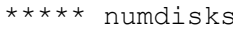

$\star \star \star * * \operatorname{NPCM}()$

$\star \star \star \star \star$ ncount

$\star \star \star \star *$ nskip

$\star \star \star * *$ offset ()

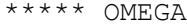

$\star \star * * *$ outfil

$\star \star \star \star *$ pchan ()

counter used to read binary data stored in unix format

name of input file

required when reading records stored in unix format

record of integer count values to be saved as output $* \star \star * *$

Number of channels to convert-190 measured, 5 time $\quad * \star \star * *$

Number of channels representing pressure taps $\star \star \star \star \star * *$

number of disks to be processed

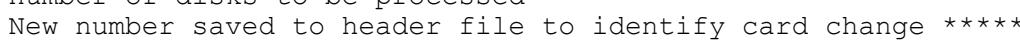

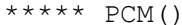

$\star \star \star \star \star * ~ \mathrm{q}$

$\star \star \star \star \star *$ radius ()

$\star \star \star * * *$

$\star * * * *$ record ()

$\star \star \star \star \star \star \operatorname{sip}()$

$\star \star \star \star \star *$ slope ()

$\star \star \star \star * *$ tempstr

$\star \star \star * \star *$ tprad $(4)$

$\star \star \star \star * *$ tpress (4)

$\star * \star * *$ windsp

$\star \star * * *$ ZERO

Number of original channels to skip in conversion process $* * *$

Array containing offset listed in header file $* * \star \star *$

rotational speed (72 r.p.m.)

name of output file

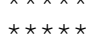

list of PCM numbers in order

dynamic pressure used for normalization

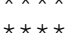

containing radii (in inches) associated with each $\star \star \star \star * *$ pressure tap by PCM number

One time step containing all channels from old E.U. file***** PCM numbers of channels to be skipped in conversion process** Array containing slope listed in header file $\quad * * * * *$ temporary string for user input

radius in inches to each total pressure probe $* * \star \star *$

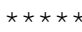

$\mathrm{PCM}$ numbers of total pressure probes $\quad * * * * *$

wind speed average over $5 \mathrm{~min}$. data set $\star_{*} * * *$

used to initialize data file with zeros

$\star \star \star \star * \star * * 10$

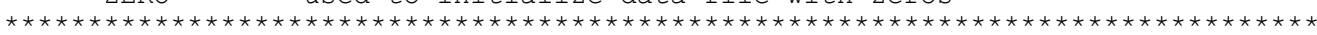

parameter (NUMCHAN=195)

parameter $($ NUMPRESS $=121)$

parameter $(\mathrm{nskip}=11)$

real slope (NUMCHAN), offset (NUMCHAN), record (239), radius (NUMPRESS)

real tprad (4)

double precision OMEGA, cforce, baro, atemp, windsp, adens, $q$

integer PCM(NUMCHAN), pchan (NUMPRESS), aoa (4), tpress (4)

integer NPCM(NUMCHAN), numdisks, disk, AZ

integer IB, test (20), h, i, j, k, m, n, SKIP (25), ncount, ITB, count

integer*2 newrec (NUMCHAN)

integer*2 cardmask(NUMCHAN) /62*16\#0000,

2

integer*2 tbit $(0: 1) / 16 \# 0000,16 \# 2000 \%$

$59 * 16 \# 8000$,

$16 * 16 \# \mathrm{C} 000 /$

integer*4 ZERO /0/

character*50 infil, outfil, testfil, header, diskstr, tempstr

character chanid(NUMCHAN) *10, disks $(1000) * 20$

character*100 instr $(239) * 4$, chan (NUMCHAN)

equivalence (instr, record)

data (aoa $(j), j=1,4) / 935,936,937,938 /$ 
data (tpress $(j), j=1,4) / 934,458,832,862 /$

data (pchan $(j), j=1$, NUMPRESS) /901,902,903,904,905,906,907,908,909, $910,911,912,913,914,915,916,917,918,919,920,921,922,923,924$, $925,926,927,928,929,930,931,932,933,427,428,429,430,431,432$, $433,434,435,436,437,438,439,440,441,442,443,444,445,446,447$, $448,449,450,451,452,453,454,455,456,457,804,805,806,807,808$, $809,810,811,812,813,814,815,816,817,818,819,820,821,822,823$, $824,825,826,827,828,829,830,831,833,834,835,836,837,838,839$, $840,841,842,843,844,845,846,847,848,849,850,851,852,853,854$, $855,856,857,858,859,860,861 /$

data (radius $(j), j=1, \operatorname{NUMPRESS}) / 103.4,103.4,125.3,125.3,125.3,125.3$, $125.3,125.3,125.3,125.3,125.3,125.3,125.3,125.3,125.3,125.3$, $125.3,125.3,125.3,125.3,125.3,125.3,125.3,125.3,125.3,125.3$, $125.3,125.3,125.3,125.3,114.4,136.4,114.4,158.4,158.4,158.4$, $158.4,158.4,158.4,158.4,158.4,158.4,158.4,158.4,158.4,158.4$, $158.4,158.4,158.4,158.4,158.4,158.4,158.4,158.4,158.4,158.4$, $158.4,158.4,158.4,158.4,158.4,147.3,147.3,136.4,59.4,59.4$, $59.4,59.4,59.4,59.4,59.4,59.4,59.4,59.4,59.4,59.4,59.4,59.4$, $59.4,59.4,59.4,59.4,59.4,59.4,59.4,59.4,59.4,59.4,59.4,70.5$, $70.5,81.4,92.3,92.3,92.3,92.3,92.3,92.3,92.3,92.3,92.3,92.3$, $92 \cdot 3,92.3,92 \cdot 3,92 \cdot 3,92 \cdot 3,92 \cdot 3,92.3,92 \cdot 3,92 \cdot 3,92 \cdot 3,92 \cdot 3,92.3$, $92.3,92.3,92.3,92.3,92.3,92.3,81.4 /$

data $(\operatorname{tprad}(j), j=1,4) / 133.3,170.9,67.4,100.3 /$

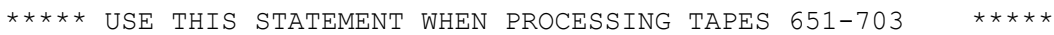
$\operatorname{data}(\operatorname{SKIP}(j), j=1, \operatorname{nskip}) / 310,311,409,108,314,315,316,415,417$, $2 \quad 107,937$

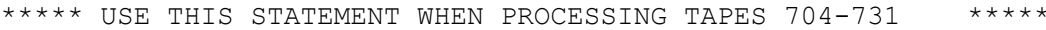
C data(SKIP (j) $, j=1, n s k i p) / 310,311,409,108,415,937,314,315,316 /$

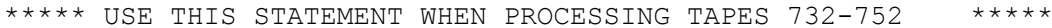

C $\quad \operatorname{data}(\operatorname{SKIP}(j), j=1, \operatorname{nskip}) / 310,311,409,108,937 /$

$\mathrm{AZ}=57$

chan (1)="Pressure\#11, 52\% span, 36\% upper""," "Cp" chan $(2)=$ "Pressure\#18, 52\% span, 4\% upper" ", "Cp" chan (3) ="Pressure\#1, 63\% span, trailing""," "Cp" chan $(4)=$ "Pressure\#2, 63\% span, 92\% upper""," "Cp" chan (5)="Pressure\#4, 63\% span, 80\% upper""," "Cp" chan (6) ="Pressure\#6, 63\% span, 68\% upper""," "Cp" chan $(7)=$ "Pressure\#8, 63\% span, 56\% upper"", "Cp" chan (8) ="Pressure\#10, 63\% span, 44\% upper""," "Cp" chan $(9)=$ "Pressure\#11, 63\% span, 36\% upper""," "Cp" chan $(10)=$ "Pressure\#12, 63\% span, 28\% upper"",""Cp" chan (11)="Pressure\#13, 63\% span, 20\% upper""," "Cp" chan (12)="Pressure\#14, 63\% span, 14\% upper""," "Cp" chan (13) ="Pressure\#15, 63\% span, 10\% upper""," "Cp" chan (14)="Pressure\#16, 63\% span, 8\% upper""," "Cp" chan $(15)=$ "Pressure\#17, 63\% span, 6\% upper""," Cp" chan (16) ="Pressure\#18, 63\% span, 4\% upper""," "Cp" chan $(17)=$ "Pressure\#19, 63\% span, 2\% upper""," "Cp" chan (18) ="Pressure\#20, 63\% span, 1\% upper""," "Cp" chan (19) ="Pressure\#21, 63\% span, 0.5\% upper""," "Cp" chan $(20)=$ "Pressure\#22, 63\% span, 0\% leading""," "Cp" chan $(21)=$ "Pressure\#23, 63\% span, 0.5\% lower" " "Cp" chan (22)="Pressure\#24, 63\% span, 1\% lower"", "Cp" chan (23) ="Pressure\#25, 63\% span, 2\% lower""," "Cp" chan $(24)=$ "Pressure\#26, 63\% span, 4\% lower"", "Cp" chan (25)="Pressure\#28, 63\% span, 8\% lower""," "Cp" chan (26) ="Pressure\#30, 63\% span, 14\% lower""," "Cp" chan (27) ="Pressure\#32, 63\% span, 28\% lower""," "Cp" chan (28) ="Pressure\#34, 63\% span, 44\% lower""," "Cp" chan $(29)=$ "Pressure\#36, 63\% span, 68\% lower""," "Cp" chan (30) ="Pressure\#38, 63\% span, 92\% lower""," "Cp" chan $(31)=$ "Pressure\#18, 58\% span, 4\% upper""," "Cp" chan (32) ="Pressure\#18, 69\% span, 4\% upper""," Cp" chan (33) ="Pressure\#11, 58\% span, 36\% upper""," "Cp" chan (34)="Total Pressure Probe, 67\% span"","Pascal" chan (35)="Digital 80\% LFA"",""deg" chan (36) ="Digital 63\% LFA"",""deg" chan (37) ="Digital 47\% LFA""," "deg" chan (38)="Digital 30\% LFA""," "deg" chan (39) ="VPA Prop Vane Speed WS-1 (12:00)""," "mps" chan $(40)=$ "VPA Prop Vane Speed WS-2 ( 1:30)"",""mps" 
chan $(41)=$ "VPA Prop Vane Speed WS-3 ( $3: 00) " ", "$ mps" chan (42)="VPA Prop Vane Speed WS-4 ( 4:30)""," "mps" chan (43)="VPA Prop Vane Speed WS-5 ( 6:00)"",""mps" chan (44)="VPA Prop Vane Speed WS-6 ( 7:30)""," "mps" chan (45)="VPA Prop Vane Speed WS-7 (9:00)"","mps" chan (46)="VPA Prop Vane Speed WS-8 (10:30)""," "mps" chan (47)="VPA Prop Vane Speed WS-9 Hub Height"","mps" chan (48)="VPA Prop Vane Direction WD-9 Hub Height""," "deg" chan (49)="VPA Bi-Vane speed WS-12 (3:00 @100\%)""," "mps" chan (50)="VPA Bi-Vane Direction WD-12 (3:00 @100\%)""," "deg" chan (51)="VPA Bi-Vane Elevation WE-12 (3:00 @100\%)"",""deg" chan (52)="VPA Bi-Vane speed WS-13 (9:00 @100\%)""," "mps" chan (53)="VPA Bi-Vane Direction WD-13 (9:00 @100\%)"", "deg" chan (54)="VPA Bi-Vane Elevation WE-13 (9:00 @100\%)"",""deg" chan (55)="VPA Prop Vane Speed WS-10 (12:00 @40\%)""," "mps" chan (56)="VPA Prop Vane Speed WS-11 (6:00 @40\%)""," "mps" chan $(57)=$ "Low Speed Shaft Azimuth Angle"",""deg" chan (58) ="Yaw Moment"", "N-m"

chan (59)="Tower Bending about East-West Axis (X)"",""N-m" chan $(60)=$ "Tower Bending about North-South Axis (Y) "", "N-m" chan $(61)=$ "Yaw Angle""," "deg"

chan (62) ="Generator Power""," "kW"

chan (63) ="TSI (South) X-Film X (Not Working)"","Volt" chan (64)="TSI (South) X-Film Y (Not Working)"","Volt" chan (65)="Sonic Anemometer Channel A "",""mps" chan (66)="Sonic Anemometer Channel B "",""mps" chan (67)="Sonic Anemometer Channel C "","mps" chan (68)="Strain Blade 3A, root flap bending"",""N-m" chan (69)="Strain Blade 3B, root flap bending"", "N-m" chan $(70)=$ "Strain Blade 1, root flap bending""," "N-m" chan $(71)=$ "Strain Blade 2, root flap bending""," "N-m" chan (72)="Strain Blade 3, 20\% flap bending"", "N-m" chan (73) ="Strain Blade 3, 40\% flap bending"", "N-m" chan $(74)=$ "Strain Blade 3, 50\% flap bending""," "N-m" chan (75)="Strain Blade 3, 70\% flap bending"", "N-m" chan (76) ="Strain Blade 3, 90\% flap bending"", "N-m" chan (77)="Strain Blade 3, root edge bending""," "N-m" chan (78)="Strain Blade 3, 20\% edge bending"", "N-m" chan $(79)=$ "Strain Blade 3, 50\% edge bending"", "N-m" chan $(80)=" 70 \%$ blade torque" ", "N-m"

chan $(81)=$ "Root torque (link)" ", " "N-m"

chan $(82)=" B l a d e ~ 3$ Torsion at 50\% Span""," "N-m"

chan (83) ="Strain X-X LSS bending""," "N-m"

chan $(84)=$ "Strain $Y-Y$ LSS bending"", "N-m"

chan (85)="Strain LSS torque A""," "N-m"

chan $(86)=$ "Strain LSS torque B"", "N-m"

chan $(87)=$ "Pressure\#1, 80\% span, trailing""," "Cp" chan (88) ="Pressure\#2, 80\% span, 92\% upper""," "Cp" chan (89) ="Pressure\#4, 80\% span, 80\% upper""," "Cp" chan $(90)=$ "Pressure\#6, 80\% span, 68\% upper""," "Cp" chan $(91)=$ "Pressure\# 8, 80\% span, 56\% upper"", "Cp" chan (92) ="Pressure\#10, 80\% span, 44\% upper""," "Cp" chan (93) ="Pressure\#11, 80\% span, 36\% upper"", "Cp" chan $(94)=$ "Pressure\#12, 80\% span, 28\% upper""," "Cp" chan (95) ="Pressure\#13, 80\% span, 20\% upper""," "Cp" chan (96) ="Pressure\#14, 80\% span, 14\% upper""," "Cp" chan $(97)=$ "Pressure\#15, 80\% span, 10\% upper""," $\mathrm{Cp} "$ chan (98)="Pressure\#16, $80 \%$ span, 8\% upper" ", "Cp" chan (99) ="Pressure\#17, 80\% span, 6\% upper""," "Cp" chan $(100)=$ "Pressure\#18, 80\% span, 4\% upper""," "Cp" chan $(101)=$ "Pressure\#19, 80\% span, 2\% upper""," "Cp" chan $(102)=$ "Pressure\#20, 80\% span, 1\% upper" " " "Cp" chan $(103)=$ "Pressure\#21, 80\% span, 0.5\% upper""," "Cp" chan $(104)=$ "Pressure\#22, 80\% span, 0\% leading""," "Cp" chan (105) ="Pressure\#23, 80\% span, 0.5\% lower""," "Cp" chan (106) ="Pressure\#24, 80\% span, 1\% lower"",""Cp" chan (107) ="Pressure\#25, 80\% span, 2\% lower""," Cp" chan $(108)=$ "Pressure\#26, 80\% span, 4\% lower""," "Cp" chan (109) ="Pressure\#28, 80\% span, 8\% lower"",""Cp" chan $(110)=$ "Pressure\#30, 80\% span, 14\% lower""," "Cp" chan $(111)=$ "Pressure\#32, 80\% span, 28\% lower""," "Cp" chan $(112)=$ "Pressure\#34, 80\% span, 44\% lower""," "Cp" chan (113) ="Pressure\#36, 80\% span, 68\% lower""," "Cp" chan $(114)=$ "Pressure\#38, 80\% span, 92\% lower""," "Cp" chan $(115)=$ "Pressure\#18, $74 \%$ span, 4\% upper"", "Cp" chan $(116)=$ "Pressure\#11, 74\% span, 36\% upper"",""Cp" 
chan $(117)=$ "Pressure\#11, 69\% span, 36\% upper""," Cp" chan $(118)=$ "Total pressure probe, 86\% span"" "Pascal" chan (119) ="Absolute reference pressure""," Pa" chan (120) ="Blade 3 Pitch angle""," "deg" chan $(121)=$ "Pressure\#1, 30\% span, trailing""," "Cp" chan (122) ="Pressure\#4, 30\% span, 80\% upper""," "Cp" chan (123) ="Pressure\#6, 30\% span, 68\% upper""," "Cp" chan (124) ="Pressure\# 8, 30\% span, 56\% upper""," "Cp" chan $(125)=$ "Pressure\#10, 30\% span, 44\% upper""," "Cp" chan $(126)=$ "Pressure\#11, 30\% span, 36\% upper""," "Cp" chan (127) ="Pressure\#13, 30\% span, 20\% upper""," "Cp" chan $(128)=$ "Pressure\#14, 30\% span, 14\% upper""," "Cp" chan (129) ="Pressure\#15, 30\% span, 10\% upper" ","Cp" chan $(130)=$ "Pressure\#16, 30\% span, 8\% upper""," "Cp" chan (131) ="Pressure\#17, 30\% span, 6\% upper""," "Cp" chan (132) ="Pressure\#18, 30\% span, 4\% upper""," "Cp" chan (133) ="Pressure\#19, 30\% span, 2\% upper"",""Cp" chan $(134)=$ "Pressure\#21, 30\% span, 0.5\% upper""," $\mathrm{Cp} "$ chan (135) ="Pressure\#22, 30\% span, 0\% leading""," "Cp" chan $(136)=$ "Pressure\#23, 30\% span, $0.5 \%$ lower"", "Cp" chan $(137)=$ "Pressure\#24, 30\% span, 1\% lower" " " "Cp" chan (138) ="Pressure\#25, 30\% span, 2\% lower""," "Cp" chan (139) ="Pressure\#26, 30\% span, 4\% lower""," "Cp" chan $(140)=$ "Pressure\#28, 30\% span, 8\% lower""," "Cp" chan (141) ="Pressure\#30, 30\% span, 14\% lower""," "Cp" chan (142)="Pressure\#31, 30\% span, 20\% lower""," "Cp" chan (143) ="Pressure\#34, 30\% span, 44\% lower""," "Cp" chan $(144)=$ "Pressure\#36, 30\% span, 68\% lower""," "Cp" chan (145) ="Pressure\#38, 30\% span, 92\% lower""," "Cp" chan (146) ="Pressure\#18, 36\% span, 4\% upper""," "Cp" chan $(147)=$ "Pressure\#11, 36\% span, 36\% upper""," "Cp" chan $(148)=$ "Pressure\#11, 41\% span, 36\% upper""," "Cp" chan (149)="Total pressure probe, 34\% span""," Pascal" chan $(150)=$ "Pressure\#1, 47\% span, trailing""," $\mathrm{Cp} "$ chan $(151)=$ "Pressure\#2, 47\% span, 92\% upper""," "Cp" chan $(152)=$ "Pressure\#4, 47\% span, 80\% upper""," "Cp" chan (153) ="Pressure\#6, 47\% span, 68\% upper""," "Cp" chan $(154)=$ "Pressure\#8, 47\% span, 56\% upper""," "Cp" chan (155) ="Pressure\#10, 47\% span, 44\% upper""," "Cp" chan $(156)=$ "Pressure\#11, 47\% span, 36\% upper""," "Cp" chan $(157)=$ "Pressure\#12, 47\% span, 28\% upper"",""Cp" chan $(158)=$ "Pressure\#13, 47\% span, 20\% upper""," "Cp" chan (159) ="Pressure\#14, 47\% span, 14\% upper""," "Cp" chan $(160)=$ "Pressure\#15, 47\% span, 10\% upper"",""Cp" chan $(161)=$ "Pressure\#16, 47\% span, 8\% upper""," "Cp" chan $(162)=$ "Pressure\#17, 47\% span, 6\% upper""," "Cp" chan (163) ="Pressure\#18, 47\% span, 4\% upper""," "Cp" chan (164) ="Pressure\#19, 47\% span, 2\% upper""," "Cp" chan $(165)=$ "Pressure\#20, 47\% span, 1\% upper""," "Cp" chan $(166)=$ "Pressure\#21, 47\% span, 0.5\% upper""," "Cp" chan $(167)=$ "Pressure\#22, 47\% span, 0\% leading""," "Cp" chan (168) ="Pressure\#23, 47\% span, 0.5\% lower""," "Cp" chan (169) ="Pressure\#25, 47\% span, 2\% lower""," "Cp" chan $(170)=$ "Pressure\#26, 47\% span, 4\% lower""," "Cp" chan $(171)=$ "Pressure\#27, 47\% span, 6\% lower"",""Cp" chan (172) ="Pressure\#28, 47\% span, 8\% lower""," "Cp" chan (173) ="Pressure\#30, 47\% span, 14\% lower""," "Cp" chan $(174)=$ "Pressure\#32, 47\% span, 28\% lower""," "Cp" chan $(175)=$ "Pressure\#34, 47\% span, 44\% lower""," "Cp" chan $(176)=$ "Pressure\#36, 47\% span, 68\% lower""," "Cp" chan $(177)=$ "Pressure\#38, 47\% span, 92\% lower"",""Cp" chan (178) ="Pressure\#18, 41\% span, 4\% upper""," "Cp" chan (179)="Total pressure probe, 51\% span"","Pascal" chan $(180)=$ "North met WD $5 \mathrm{~m} "$ ", " "deg" chan $(181)=$ "North met WS 5 m""," "mps" chan (182) ="North met WD $10 \mathrm{~m} "$ "," "deg" chan $(183)=$ "North met WS $10 \mathrm{~m}$ (Not working)" ", "mps" chan (184)="North met WD 20 m""," "deg" chan $(185)=$ "North met WS 20 m""," "mps" chan $(186)=$ "North met WD $50 \mathrm{~m} "$ ", "deg" chan (187) ="North met WS 50 m""," "mps" chan $(188)=$ "North met Temperature $5 \mathrm{~m} "$ "," "degC" chan $(189)=$ "North met Delta Temperature T50-T05"", "degC" chan (190)="Barometric Pressure""," "Pascal" chan (191)="Time-day" ", "DAY" chan (192)="Time-hour"", "HOUR" 
chan (193)="Time-minute"", " "MINUTE"

chan (194) ="Time-second""," "SECOND"

chan (195)="Time-millisecond""," "MSEC"

$\star \star \star \star *$ PROMPT THE USER FOR THE NAME OF THE INPUT FILE NAME $* \star * \star *$

$\star \star \star \star *$ IF THE FILE EXTENSION IS '.lst', A LIST OF DISKS IS ASSUMED $* * \star \star *$

60 print*

print*, 'Enter the input file name (no extension) [',

2 infil(1:((len trim(infil)) -4)), ']'

write $(6,4)$

4 format(' Hit ENTER to accept the default: ',\$)

read' (a50)', tempstr

if (len_trim(tempstr) .gt. 0) then

2 if (tempstr( (len

diskstr=tempstr (1: len_trim(tempstr))

open (unit=13, file=diskstr, iostat=inerr, status='old')

if (inerr. ne. 0) then

2

print*,'File ', tempstr(1:len_trim(tempstr)),

' does not exist. Try again.

goto 60

endif

$m=1$

$76 \operatorname{read}\left(13,{ }^{\prime}(\mathrm{a})\right.$ ', end=77) disks $(\mathrm{m})$

print*, disks (m)

$$
\mathrm{m}=\mathrm{m}+1
$$

goto 76

77 numdisks=m-1

print*, numdisks

close (13)

infil = disks(1) (1:len trim(disks(1)))//'.dat'

header $=$ infil $\left(1:\left(\left(\right.\right.\right.$ len_trim $\left.\left.\left.\left._{\text {infil }}\right)\right)-4\right)\right) / /$ '.hdr'

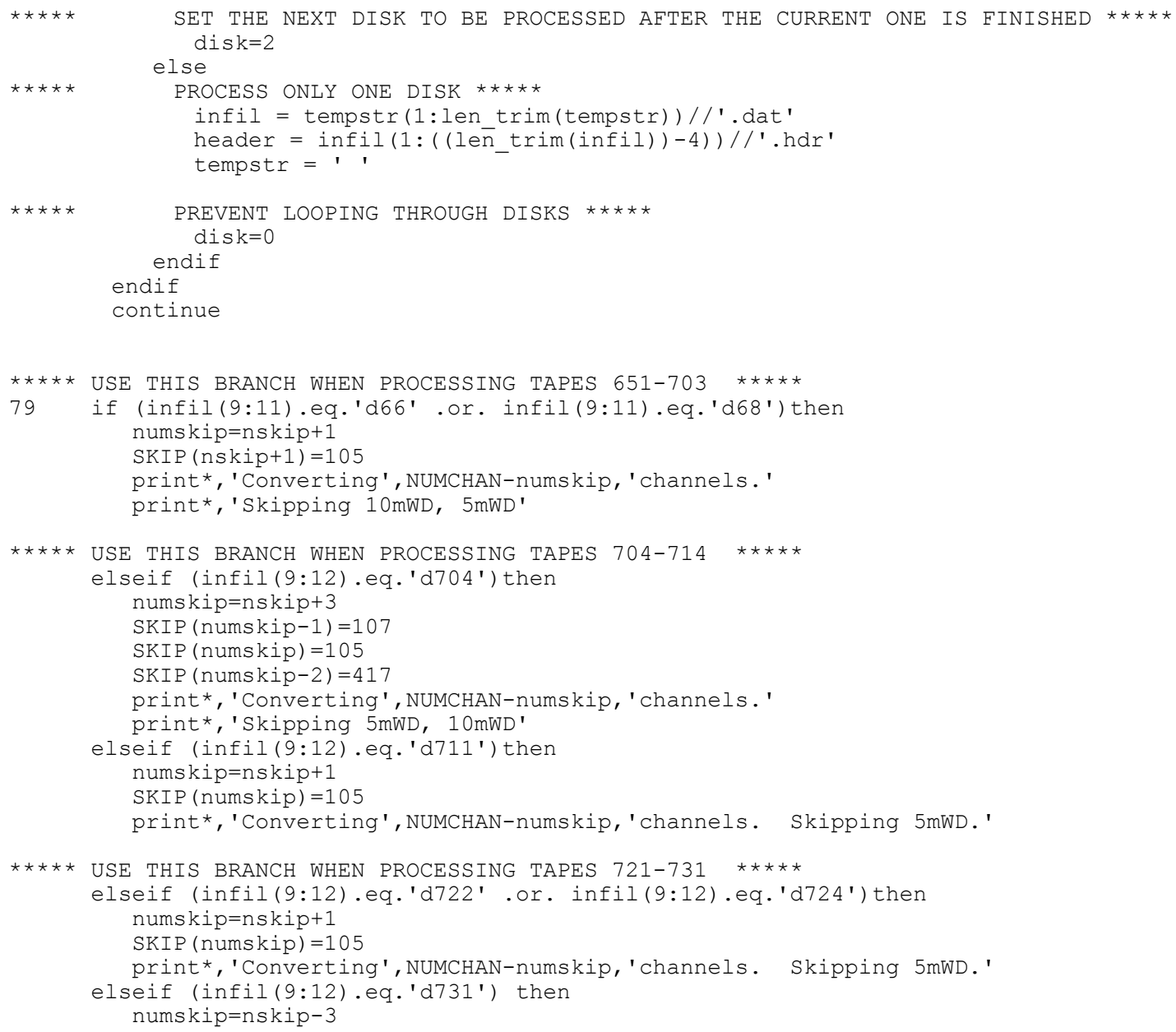




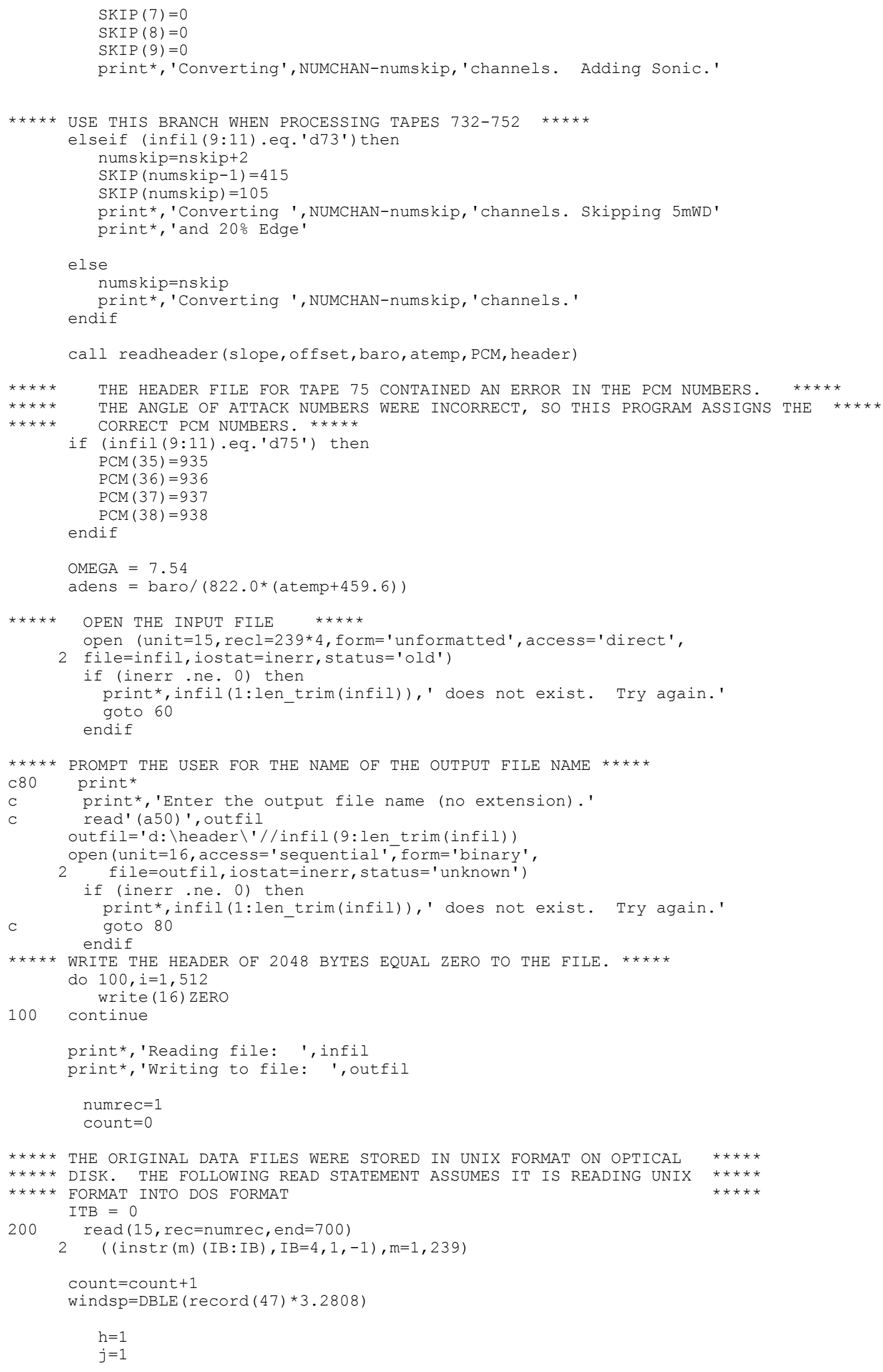




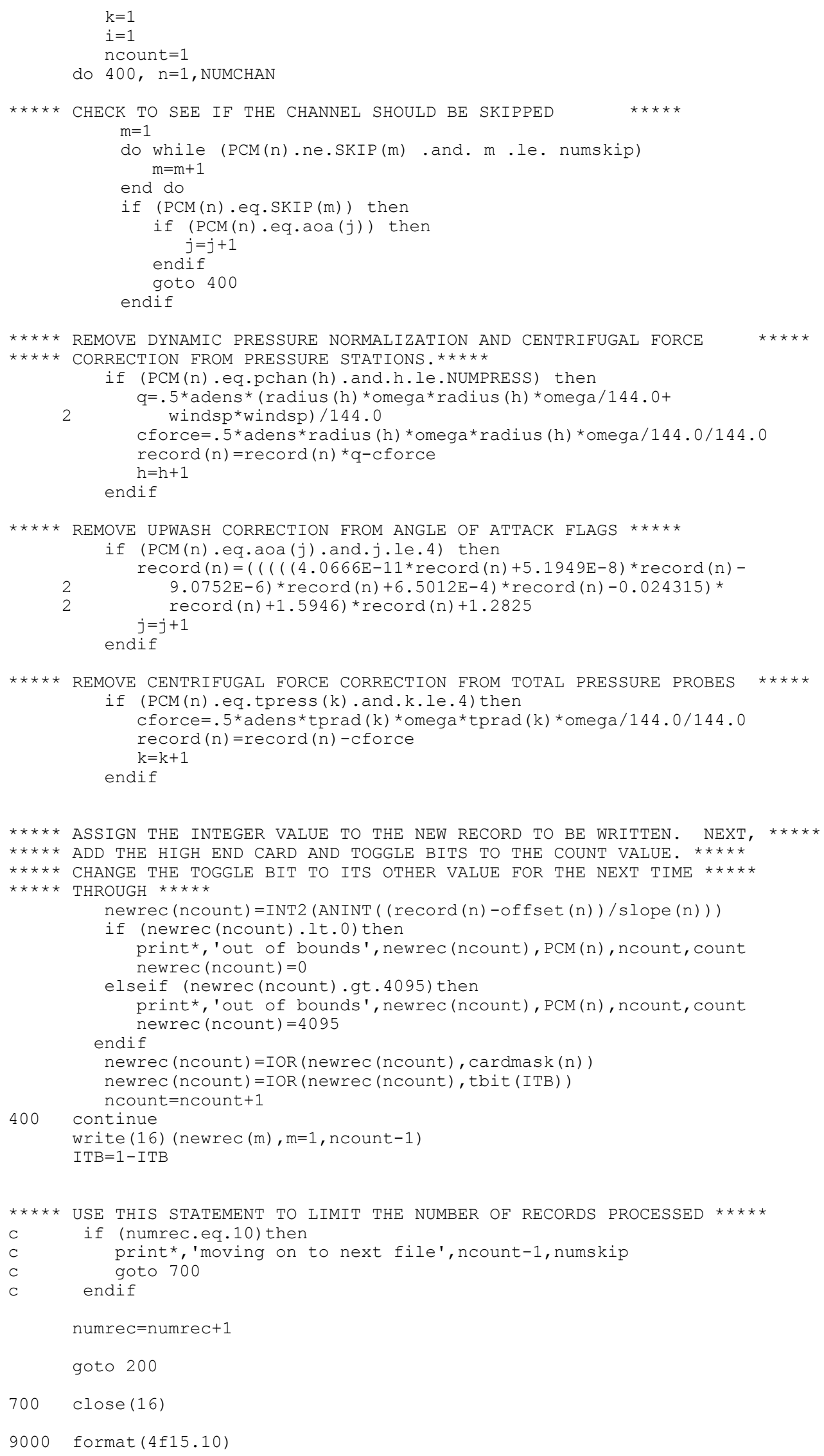




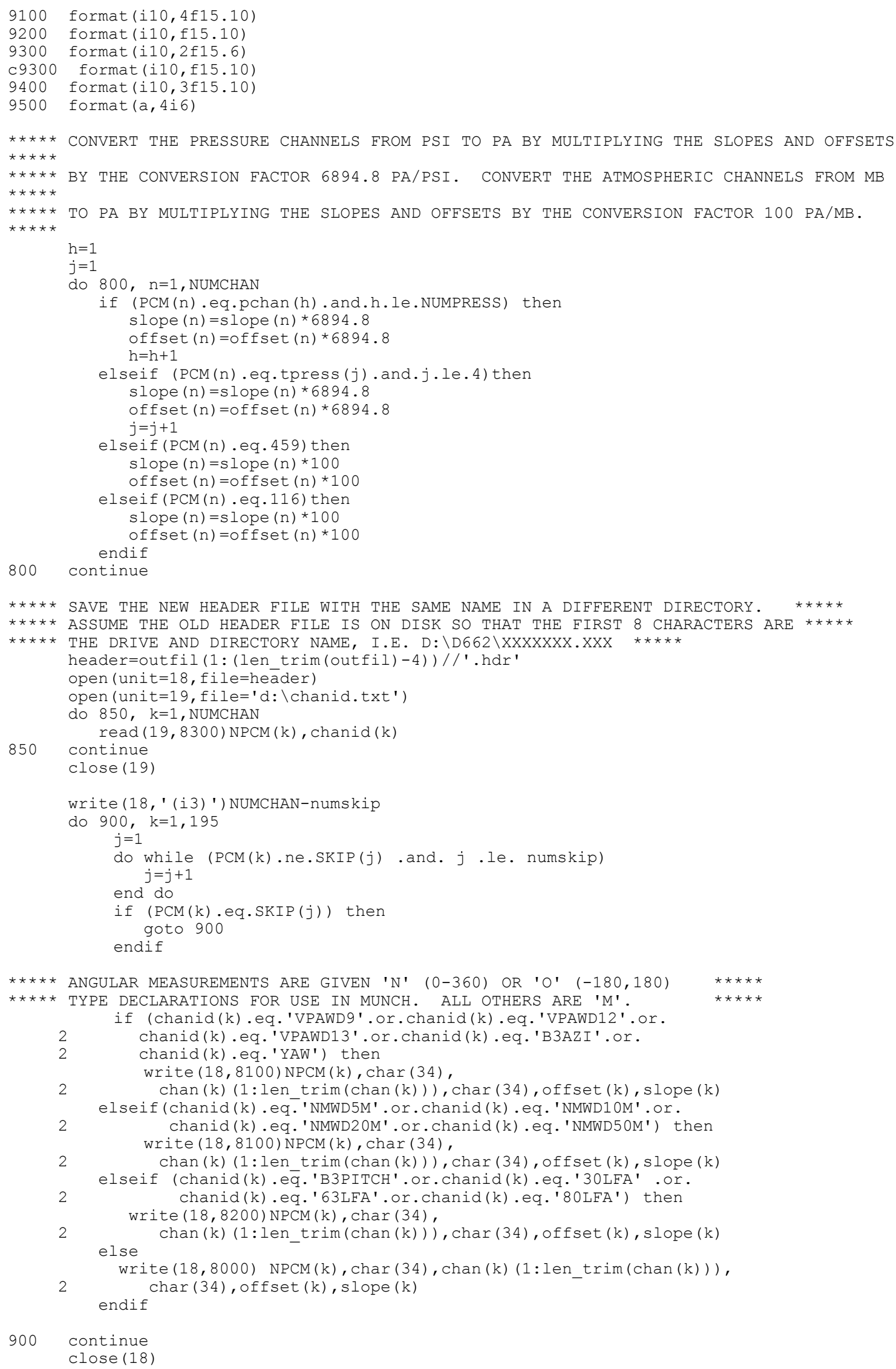




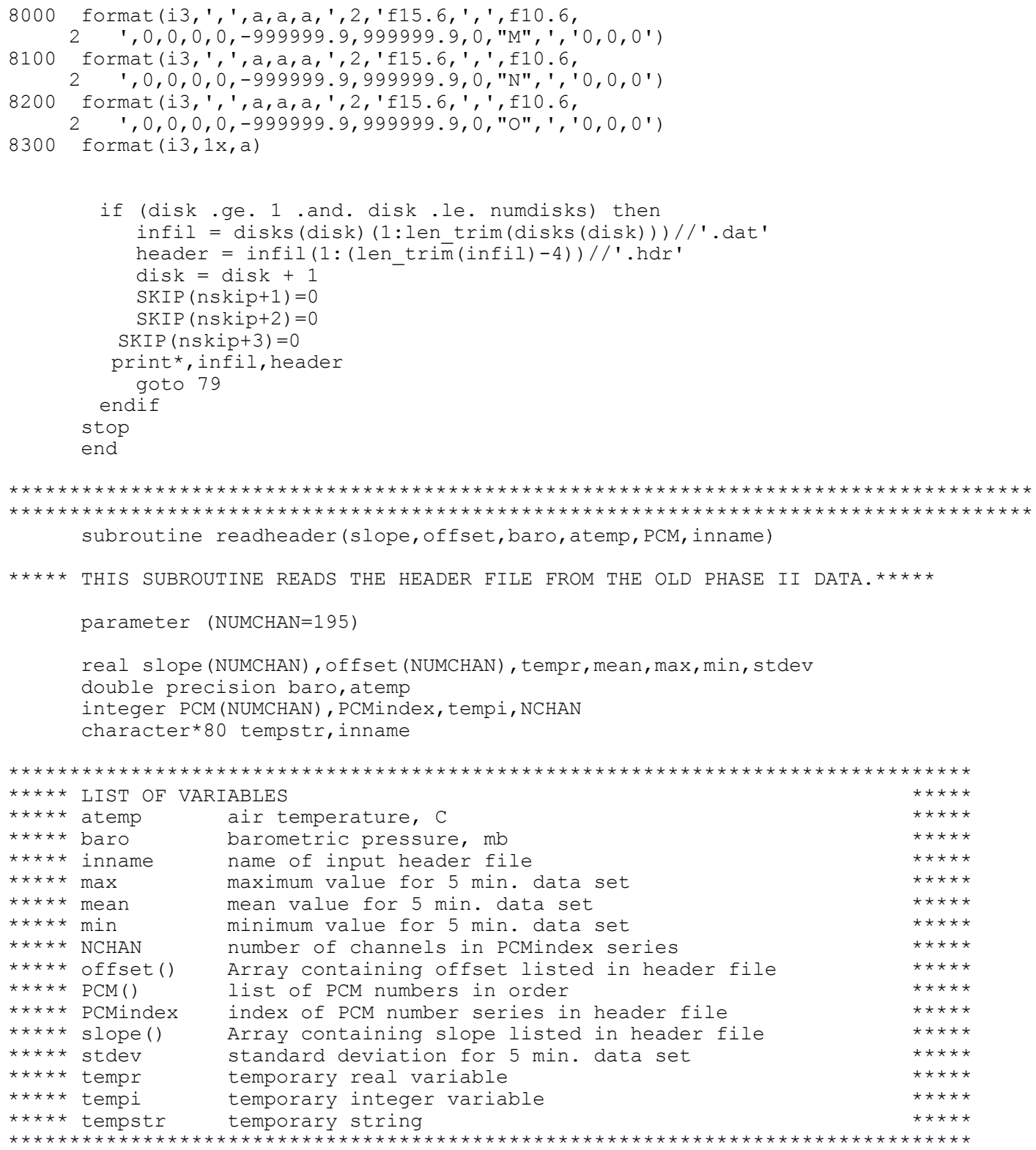

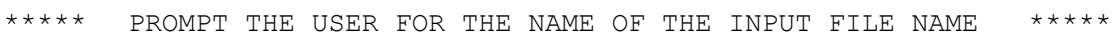




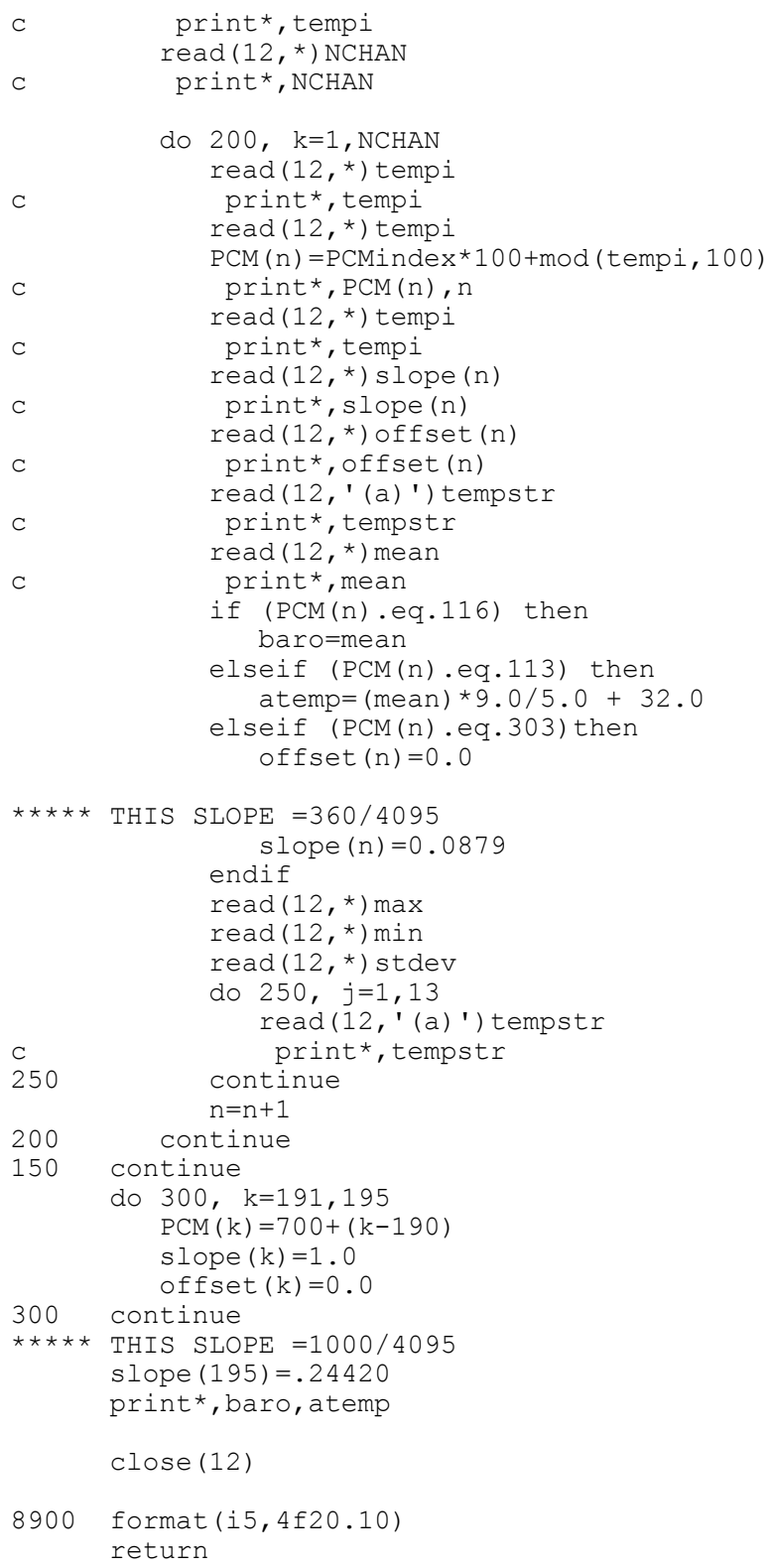




\section{Appendix B \\ Comparison of Channels on a Sample by Sample Basis}




\begin{tabular}{|c|c|c|c|c|c|}
\hline Channel & $\begin{array}{c}\text { New Value } \\
\text { at Maximum }\end{array}$ & $\begin{array}{l}\text { Old Value at } \\
\text { Maximum }\end{array}$ & $\begin{array}{c}\text { Difference } \\
\text { at Maximum }\end{array}$ & $\begin{array}{c}\text { Maximum \% } \\
\text { Difference }\end{array}$ & $\begin{array}{l}\text { No. Times } \\
\text { Difference } \\
\text { Exceeded }\end{array}$ \\
\hline TP67 & 269.8412 & 301.3969 & 31.5557 & 10 & 154124 \\
\hline $80 \mathrm{AOA}$ & -0.0172 & 0.0104 & 0.0276 & 266 & 153219 \\
\hline $63 \mathrm{AOA}$ & $\begin{array}{l}-0.0229 \\
\end{array}$ & 0.0101 & 0.0330 & 326 & 154438 \\
\hline 30AOA & -0.0253 & 0.0024 & 0.0277 & 1139 & 143210 \\
\hline VPAWS1 & 0.0000 & 0.0000 & 0.0000 & -9999999 & 0 \\
\hline VPAWS2 & 0.0000 & 0.0000 & 0.0000 & -9999999 & 0 \\
\hline VPAWS3 & 0.0000 & 0.0000 & 0.0000 & -9999999 & 0 \\
\hline VPAWS4 & 0.0000 & 0.0000 & 0.0000 & -9999999 & 0 \\
\hline VPAWS5 & 0.0000 & 0.0000 & 0.0000 & -9999999 & 0 \\
\hline VPAWS6 & 0.0000 & 0.0000 & 0.0000 & -9999999 & 0 \\
\hline VPAWS7 & 0.0000 & 0.0000 & 0.0000 & -9999999 & 0 \\
\hline VPAWS8 & 0.0000 & 0.0000 & 0.0000 & -9999999 & 0 \\
\hline VPAWS9 & 0.0000 & 0.0000 & 0.0000 & $\begin{array}{l}-9999999 \\
\end{array}$ & 0 \\
\hline VPAWD9 & 0.0000 & 0.0000 & 0.0000 & -9999999 & 0 \\
\hline VPAWS12 & 0.0000 & 0.0000 & 0.0000 & -9999999 & 0 \\
\hline VPAWD12 & 0.0000 & 0.0000 & 0.0000 & -9999999 & 0 \\
\hline VPAWA12 & 0.0060 & 0.0068 & 0.0008 & 12 & 41039 \\
\hline VPAWS13 & 0.0000 & 0.0000 & 0.0000 & -9999999 & 0 \\
\hline VPAWD13 & 0.0000 & 0.0000 & 0.0000 & -9999999 & 0 \\
\hline VPAWA13 & 0.0013 & 0.0006 & -0.0007 & 117 & 17399 \\
\hline VPAWS10 & 0.0000 & 0.0000 & 0.0000 & -9999999 & 0 \\
\hline VPAWS11 & 0.0000 & 0.0000 & 0.0000 & -9999999 & 0 \\
\hline B3AZI & 0.0000 & 0.0377 & 0.0377 & 100 & 39657 \\
\hline YAWMOM & 0.0000 & 0.0000 & 0.0000 & -9999999 & 0 \\
\hline TBEWAX & 0.0000 & 0.0000 & 0.0000 & -9999999 & 0 \\
\hline TBNSAY & 0.0000 & 0.0000 & 0.0000 & -9999999 & 0 \\
\hline YAW & 0.0000 & 0.0000 & 0.0000 & -9999999 & 0 \\
\hline GENPOW & 0.0083 & 0.0083 & 0.0000 & 0 & 55 \\
\hline B1ARFB & 0.9479 & 0.9471 & -0.0008 & 0 & 855 \\
\hline B1BRFB & 0.2920 & 0.2919 & -0.0001 & 0 & 196 \\
\hline B2RFB & 0.1126 & 0.1128 & 0.0002 & 0 & 170 \\
\hline B3RFB & 0.6064 & 0.6058 & -0.0006 & 0 & 648 \\
\hline $\mathrm{B} 120 \mathrm{FB}$ & 0.0396 & 0.0392 & -0.0003 & 1 & 273 \\
\hline B140FB & 0.4484 & 0.4478 & -0.0006 & 0 & 1300 \\
\hline $\mathrm{B} 150 \mathrm{FB}$ & 0.5232 & 0.5238 & 0.0006 & 0 & 2120 \\
\hline B170FB & 0.1965 & 0.1961 & -0.0003 & 0 & 2764 \\
\hline B190FB & 0.0537 & 0.0528 & -0.0009 & 2 & 66701 \\
\hline B1AREB & 0.3656 & 0.3665 & 0.0009 & 0 & 381 \\
\hline B120EB & 1.3417 & 1.3412 & -0.0006 & 0 & 286 \\
\hline B150EB & 1.1064 & 1.1057 & -0.0006 & 0 & 1031 \\
\hline 70TQ & 0.0000 & 0.0000 & 0.0000 & -9999999 & 0 \\
\hline RTTQ & 0.0390 & 0.0389 & -0.0001 & 0 & 2596 \\
\hline 50TSN & 0.1137 & 0.1133 & -0.0004 & 0 & 4414 \\
\hline LSSXXB & 1.3948 & 1.3965 & 0.0017 & 0 & 89 \\
\hline LSSYYB & 0.8380 & 0.8404 & 0.0024 & 0 & 259 \\
\hline LSSTQA & 2.1682 & 2.1690 & 0.0008 & 0 & 4 \\
\hline LSSTQB & 1.5952 & 1.5944 & -0.0008 & 0 & 6 \\
\hline
\end{tabular}




\begin{tabular}{|c|c|c|c|c|c|}
\hline Channel & $\begin{array}{c}\text { New Value } \\
\text { at Maximum }\end{array}$ & $\begin{array}{c}\text { Old Value at } \\
\text { Maximum }\end{array}$ & $\begin{array}{c}\text { Difference } \\
\text { at Maximum }\end{array}$ & $\begin{array}{c}\text { Maximum \% } \\
\text { Difference }\end{array}$ & $\begin{array}{l}\text { No. Times } \\
\text { Difference } \\
\text { Exceeded }\end{array}$ \\
\hline TP86 & 500.2993 & 461.0866 & -39.2127 & 9 & 154303 \\
\hline ABSRP & 0.0000 & 0.0000 & 0.0000 & -9999999 & 0 \\
\hline B1PITCH & 0.0000 & 0.0000 & 0.0000 & -9999999 & 0 \\
\hline TP34 & 0.1865 & 0.4621 & 0.2755 & 60 & 151541 \\
\hline TP51 & -2.3864 & 2.5815 & 4.9679 & 192 & 155988 \\
\hline NMWD5M & 0.0000 & 0.0000 & 0.0000 & -9999999 & 0 \\
\hline NMWS5M & 0.0000 & 0.0000 & 0.0000 & -9999999 & 0 \\
\hline NMWD10M & 0.0000 & 0.0000 & 0.0000 & -9999999 & 0 \\
\hline NMWD20M & 0.0000 & 0.0000 & 0.0000 & -9999999 & 0 \\
\hline NMWS20M & 0.0000 & 0.0000 & 0.0000 & -9999999 & 0 \\
\hline NMWD50M & 0.0000 & 0.0000 & 0.0000 & -9999999 & 0 \\
\hline NMWS50M & 0.0000 & 0.0000 & 0.0000 & -9999999 & 0 \\
\hline NMT5M & 0.0000 & 0.0000 & 0.0000 & -9999999 & 0 \\
\hline NMDT & 0.0000 & 0.0000 & 0.0000 & -9999999 & 0 \\
\hline BARO & 0.0000 & 0.0000 & 0.0000 & -9999999 & 0 \\
\hline DAY & 0.0000 & 0.0000 & 0.0000 & -9999999 & 0 \\
\hline HOUR & 0.0000 & 0.0000 & 0.0000 & -9999999 & 0 \\
\hline MINUTE & 0.0000 & 0.0000 & 0.0000 & -9999999 & 0 \\
\hline SECOND & 0.0000 & 0.0000 & 0.0000 & -9999999 & 0 \\
\hline MILLISEC & 0.2441 & 0.1600 & -0.0841 & 53 & 39578 \\
\hline
\end{tabular}


Appendix C

Comparison of Header Files 


\begin{tabular}{|l|c|r|r|}
\hline Channel & \% Difference & $\begin{array}{l}\text { Maximum } \\
\text { Difference }\end{array}$ & $\begin{array}{l}\text { Minimum } \\
\text { Difference }\end{array}$ \\
\hline TP67 & 1 & 8.7264 & 1.3572 \\
\hline 80LFA & 0 & -0.0203 & 0.1625 \\
\hline 63LFA & 0 & 0.0194 & 2.5089 \\
\hline 30LFA & 0 & -0.0342 & 1.1516 \\
\hline VPAWS1 & 0 & 0.0000 & 0.0000 \\
\hline VPAWS2 & 0 & 0.0000 & 0.0000 \\
\hline VPAWS3 & 0 & 0.0006 & 0.0003 \\
\hline VPAWS4 & 0 & 0.0000 & 0.0000 \\
\hline VPAWS5 & 0 & 0.0000 & 0.0000 \\
\hline VPAWS6 & 0 & 0.0000 & 0.0000 \\
\hline VPAWS7 & 0 & 0.0000 & 0.0000 \\
\hline VPAWS8 & 0 & 0.0000 & 0.0000 \\
\hline VPAWS9 & 0 & 0.0000 & 0.0000 \\
\hline VPAWD9 & 0 & 0.0000 & 0.0000 \\
\hline VPAWS12 & 0 & 0.0001 & 0.0000 \\
\hline VPAWD12 & 0 & 0.0003 & 0.0002 \\
\hline VPAWA12 & 0 & 0.0009 & 0.0005 \\
\hline VPAWS13 & 0 & 0.0000 & 0.0000 \\
\hline VPAWD13 & 0 & -0.0012 & -0.0010 \\
\hline VPAWA13 & 0 & -0.0009 & -0.0003 \\
\hline VPAWS10 & 0 & 0.0000 & 0.0000 \\
\hline VPAWS11 & 0 & 0.0000 & 0.0000 \\
\hline B3AZI & 0 & 0.0487 & 0.0041 \\
\hline YAWMOM & 0 & 0.0001 & 0.0001 \\
\hline TBEWAX & 0 & -0.0010 & -0.0010 \\
\hline TBNSAY & 0 & 0.0012 & 0.0009 \\
\hline YAW & 0 & 0.0000 & 0.0000 \\
\hline GENPOW & 0 & 0.0000 & 0.0000 \\
\hline B1ARFB & 0 & -0.0004 & -0.0007 \\
\hline B1BRFB & 0 & 0.0001 & 0.0001 \\
\hline B2RFB & 0 & -0.0001 & 0.0006 \\
\hline B3RFB & 0 & -0.0009 & -0.0006 \\
\hline B120FB & 0 & 0.0001 & 0.0000 \\
\hline B140FB & 0 & -0.0007 & -0.0005 \\
\hline B150FB & 0 & 0.0011 & 0.0003 \\
\hline B190FB & 0 & -0.0010 & -0.0008 \\
\hline B15REEB & 0 & 0.0012 & 0.0010 \\
\hline LSSSTSN & 0 & -0.0009 & -0.0006 \\
\hline LSSTQA & 0 & 0.0001 & -0.0001 \\
\hline
\end{tabular}




\begin{tabular}{|l|c|r|r|}
\hline Channel & \% Difference & $\begin{array}{l}\text { Maximum } \\
\text { Difference }\end{array}$ & $\begin{array}{l}\text { Minimum } \\
\text { Difference }\end{array}$ \\
\hline TP86 & 1 & 5.5052 & 5.4022 \\
\hline ABSRP & 0 & 0.0000 & 0.0001 \\
\hline B1PITCH & 0 & -0.0003 & -0.0003 \\
\hline TP34 & 0 & 1.0040 & 0.6008 \\
\hline TP51 & 2 & 2.6836 & 6.6508 \\
\hline NMWD5M & 0 & 0.0000 & 0.0000 \\
\hline NMWS5M & 0 & 0.0000 & 0.0000 \\
\hline NMWD10M & 0 & 0.0000 & 0.0000 \\
\hline NMWD20M & 0 & 0.0000 & 0.0000 \\
\hline NMWS20M & 0 & -0.0002 & -0.0001 \\
\hline NMWD50M & 0 & 0.0000 & 0.0000 \\
\hline NMWS50M & 0 & 0.0003 & 0.0000 \\
\hline NMT5M & 0 & -0.0001 & -0.0001 \\
\hline NMDT & 0 & 0.0002 & 0.0002 \\
\hline BARO & 0 & 0.0000 & 0.0000 \\
\hline
\end{tabular}




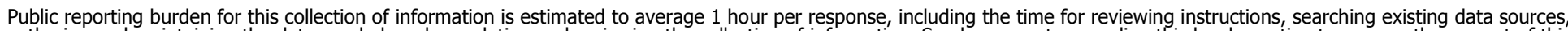

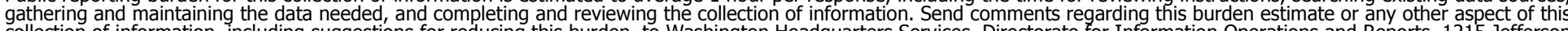

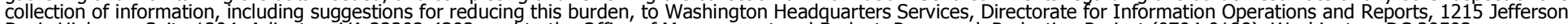

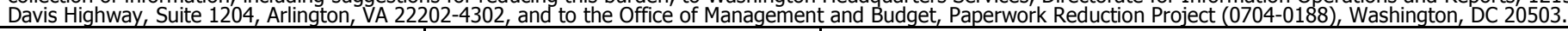

\begin{tabular}{|l|l|l|}
\hline 1. AGENCY USE ONLY (Leave blank) & $\begin{array}{c}\text { 2. REPORT DATE } \\
\text { July } 1999\end{array}$ & $\begin{array}{l}\text { 3. REPORT TYPE AND DATES COVERED } \\
\text { Technical Report }\end{array}$ \\
\hline
\end{tabular}

4. TITLE AND SUBTITLE

Conversion of Phase II Unsteady Aerodynamics Experiment Data to Common Format
5. FUNDING NUMBERS

WE901110

8. PERFORMING ORGANIZATION REPORT NUMBER

\section{AUTHOR(S) \\ M.M. Hand}

7. PERFORMING ORGANIZATION NAME(S) AND ADDRESS(ES)

National Renewable Energy Laboratory

1617 Cole Blvd.

Golden, CO 80401-3393

9. SPONSORING/MONITORING AGENCY NAME(S) AND ADDRESS(ES)

National Renewable Energy Laboratory

1617 Cole Blvd.

10. SPONSORING/MONITORING AGENCY REPORT NUMBER

TP-500-26371

11. SUPPLEMENTARY NOTES

12a. DISTRIBUTION/AVAILABILITY STATEMENT

National Technical Information Service

U.S. Department of Commerce

5285 Port Royal Road

Springfield, VA 22161

12b. DISTRIBUTION CODE

13. ABSTRACT (Maximum 200 words)

A vast amount of aerodynamic, structural, and turbine performance data were collected during three phases of the National Renewable Energy Laboratory's Unsteady Aerodynamics Experiment (UAE). To compare data from the three phases, a similar format of engineering unit data is required. The process of converting Phase II data from a previous engineering unit format to raw integer counts is discussed. The integer count files can then be input to the new post-processing software, MUNCH. The resulting Phase II engineering unit files are in a common format with current and future UAE engineering unit files. An additional objective for changing the file format was to convert the Phase II data from English units to SI units of measurement.

\section{SUBJECT TERMS}

wind energy, wind turbines, wind turbine aerodynamics

15. NUMBER OF PAGES

16. PRICE CODE

17. SECURITY CLASSIFICATION OF REPORT

18. SECURITY CLASSIFICATION OF THIS PAGE
19. SECURITY CLASSIFICATION OF ABSTRACT
20. LIMITATION OF ABSTRACT

UL 